\title{
BMJ Open Comparative efficacy and safety of surgical and invasive treatments for adults with degenerative lumbar spinal stenosis: protocol for a network meta- analysis and systematic review
}

\author{
Lingxiao Chen, ${ }^{\oplus 1}$ Paulo H Ferreira, ${ }^{2}$ Paula R Beckenkamp, ${ }^{2}$ Manuela L Ferreira ${ }^{1}$
}

To cite: Chen L, H Ferreira $P$, $\mathrm{R}$ Beckenkamp P, et al. Comparative efficacy and safety of surgical and invasive treatments for adults with degenerative lumbar spinal stenosis: protocol for a network meta-analysis and systematic review. BMJ Open 2019;9:e024752. doi:10.1136/ bmjopen-2018-024752

- Prepublication history and additional material for this paper are available online. To view these files, please visit the journal online (http://dx.doi. org/10.1136/bmjopen-2018024752).

Received 13 June 2018 Revised 14 February 2019 Accepted 15 February 2019

Check for updates

(c) Author(s) (or their employer(s)) 2019. Re-use permitted under CC BY-NC. No commercial re-use. See rights and permissions. Published by BMJ.

${ }^{1}$ Institute of Bone and Joint Research, Kolling Institute, Sydney Medical School, Faculty of Medicine and Health, University of Sydney, Sydney, New South Wales, Australia ${ }^{2}$ University of Sydney, Faculty of Health Sciences, Discipline of Physiotherapy, Sydney, New South Wales, Australia

Correspondence to

Lingxiao Chen;

Iche4036@uni.sydney.edu.au

\section{ABSTRACT}

Introduction Surgical and invasive procedures are widely used in adults with degenerative lumbar spinal stenosis when conservative treatments fail. However, little is known about the comparative efficacy and safety of these interventions. To address this, we will perform a network meta-analysis (NMA) and systematic review to compare the efficacy and safety of surgical and invasive procedures for adults with degenerative lumbar spinal stenosis. Methods and analysis We will include randomised controlled trials assessing surgical and invasive treatments for adults with degenerative lumbar spinal stenosis. We will search AMED, CINAHL, EMBASE, the Cochrane Library and MEDLINE. Only English studies will be included and no restriction will be set for publication status. For efficacy, our primary outcome will be physical function. Secondary outcomes will include pain intensity, health-related quality of life, global impression of recovery, work absenteeism and mobility. For safety, our primary outcome will be allcause mortality. Secondary outcomes will include adverse events (number of events or number of people with an event) and treatment withdrawal due to adverse effect. Two reviewers will independently select studies, extract data and assess the risk of bias (Revised Cochrane riskof-bias tool for randomized trials) of included studies. The quality of the evidence will be evaluated through the Grading of Recommendations Assessment, Development and Evaluation framework. Random-effects NMA will be performed to combine all the evidence under the frequentist framework and the ranking results will be presented through the surface under the cumulative ranking curve and mean rank. All analyses will be performed in Stata and $\mathrm{R}$.

Ethics and dissemination No ethical approval is required. The research will be published in a peerreviewed journal.

PROSPERO registration number CRD42018094180.

\section{INTRODUCTION}

Degenerative lumbar spinal canal stenosis is characterised by decreased spinal canal diameter due to structural changes of the spine (eg, facet joints, ligaments) due to ageing.
Strengths and limitations of this study

- This is the first network meta-analysis to assess the efficacy and safety of surgical and invasive procedures for adults with degenerative lumbar spinal stenosis.

- The main strengths are that only randomised controlled trials will be included for both efficacy outcomes (physical function, pain intensity, health-related quality of life, global impression of recovery, work absenteeism and mobility) and safety outcomes (all-cause mortality, adverse effect and treatment withdrawal due to adverse effect).

- Additional strength is that informative missingness difference of means for continuous outcomes and informative missing ORs for dichotomous outcomes will be used to deal with the missing data.

- The main limitation will be the limited data from lower socioeconomic countries considering the high cost of the surgical and invasive treatments.

Typically, patients will present with neurogenic claudication, defined as pain, numbness and/or fatigue in the lower limbs that is worsened during walking and standing, and alleviated with forward bending or sitting. ${ }^{12}$ In the USA, the prevalence of degenerative lumbar spinal stenosis in the general population can be as high as $22.5 \%$ for relative stenosis (ie, $\leq 12 \mathrm{~mm}$ canal diameter), and $7.3 \%$ for absolute stenosis (ie, $\leq 10 \mathrm{~mm}$ canal diameter). ${ }^{3}$ These figures increase drastically with age, reaching $47.2 \%$ and $19.4 \%$, respectively, for those 60 years of age or older. ${ }^{3}$

Most guidelines recommend a course of conservative care, including the North American Spine Society guidelines, for patients with degenerative lumbar spinal stenosis. ${ }^{2}$ However, when conservative treatments fail, surgical and invasive options are indicated. $^{245}$ Surgical decompression (including laminectomies or laminotomies), with or 
without fusion, interspinous process spacer devices, minimally invasive surgical decompression, and corticosteroidal epidural injections are commonly used in the management of spinal stenosis. ${ }^{6-11}$ However, the evidence supporting the superiority of one option over the other is still unclear for most. ${ }^{712} 13$ For instance, past meta-analyses have shown that Superion interspinous spacer is superior to X-STOP interspinous spacer in improving axial pain severity and Zurich Claudication Questionnaire (ZCQ) patient satisfaction score; whereas the addition of spinal fusion to surgical decompression does not add any benefit to surgical decompression alone. ${ }^{14} 15$ Moreover, existing meta-analyses use pairwise analytical approaches, and therefore can only provide results for the comparison of two interventions at any one time. ${ }^{411} 14-28$ A network meta-analysis (NMA) is the best design and analytical approach to compare and rank multiple interventions simultaneously, based on their relative estimate effects in each outcome. ${ }^{29}$ NMA has been used in similar fields, including sciatica, lumbar disc herniation and osteoarthritis, but, to date, no NMA has been conducted to establish the comparative effectiveness and safety of invasive approaches for degenerative lumbar spinal canal stenosis. ${ }^{30-32}$ As such, our aim is to perform an NMA and systematic review to assess the efficacy and safety of surgical and invasive procedures for adults with degenerative lumbar spinal stenosis.

\section{METHODS AND ANALYSIS}

\section{Criteria for considering studies for this review}

The protocol was written in accordance with the Preferred Reporting Items for Systematic Reviews and Meta-Analyses Protocols. ${ }^{33}$ Any changes made to this protocol will be updated in the PROSPERO registration.

\section{Types of participants}

We will include studies that recruited participants who are 40 years of age or older, with a diagnosis of degenerative lumbar spinal stenosis. We will exclude studies on patients with malignancy, trauma, vertebral fracture, infection and inflammatory disorders. For studies including degenerative lumbar spinal stenosis and associated spondylolisthesis, only those of participants with Meyerding grade I spondylolisthesis will be included. Studies including mixed populations will only be included if the data for patients with degenerative lumbar spinal stenosis can be extracted separately or if at least $80 \%$ of the patients are diagnosed with degenerative lumbar spinal stenosis.

\section{Types of interventions}

Studies comparing any surgical or invasive intervention for adults with degenerative lumbar spinal stenosis will be included. For example, surgical decompression, including laminectomies or laminotomies, with or without fusion, interspinous process spacer devices, minimally invasive surgical decompression and corticosteroidal epidural injections. The comparison group could be no treatment, usual care, sham operation, another active option or a combination of approaches. The interventions in comparison groups will be treated as different nodes. However, if we have insufficient studies to connect different interventions, we will combine no treatment and usual care into one node to make full use of the data.

\section{Outcome measures}

The outcome data will be grouped into short-term $(\leq 6$ months), mid-term (6-12 months) and long-term ( $\geq 12$ months) follow-up assessment. ${ }^{34}$ We will perform NMA in the three time points separately. For studies which report outcomes in multiple time points, data closest to the 6 and 12 months follow-up time will be included in the main analyses. For different time points in long-term follow-up assessment (eg, 1 year, 2 years, 5 years), subgroup analyses will be performed.

\section{Primary outcomes}

1. Physical function, commonly measured by Oswestry Disability Index (ODI), Roland Morris Disability Questionnaire (RMDQ), Patient-Specific Function Scale and Core Outcome Measures Index (COMI). ${ }^{34}$ Other rating scales will be included if they have been proposed in peer-reviewed journals. If the study provides more than one instruments, ODI will be used as the first choice, RMDQ as the second choice and COMI as the third choice. ${ }^{34}$

2. All-cause mortality measured by the percentage of patients who died following randomisation.

\section{Secondary outcomes}

1. Pain intensity, commonly measured by Numeric Rating Scale (NRS) and the Visual Analogue Scale (VAS). ${ }^{35} 36$ Other rating scales will also be included if they have been proposed in peer-reviewed journals. Pain intensity will be categorised and analysed according to the following three groups: back pain, leg pain and overall pain. If the study provides more than one instruments, VAS will be used as the first choice and NRS as the second choice. $^{34}$

2. Health-related quality of life, commonly measured by 36-Item Short Form Survey (SF-36), EuroQol five-dimension (EQ-5D), Nottingham health profile (NHP) and SF-12. ${ }^{34}$ SF-36, NHP and SF-12, could be mapped into EQ-5D. ${ }^{37}$ As above, other tools will also be included if they have been proposed in peer-reviewed journals. If the study provides more than one instruments, EQ-5D will be used as the first choice, following by SF36, SF-12 and NHP. ${ }^{34}$

3. Global impression of recovery measured by the percentage of the patients satisfied with their recovery.

4. Work absenteeism measured by the number of days of sick leave.

5. Mobility measured by walking distance.

6. Adverse event measured by the number of participants with an adverse event, or number of adverse events per group. Adverse events could include nerve injury, du- 
ral tear, vascular injury, deep infection and pulmonary embolus.

7. Treatment withdrawal due to adverse effect measured by the percentage of patients who drop out due to adverse effect.

\section{Types of studies}

Only randomised controlled trials, which include parallel, cross-over and cluster trials, will be included. For cross-over studies, only data before wash-out period will be used. For cluster randomised trials, we will extract data which are adjusted for clustering. If these data are unavailable, we will extract original data and adjust them for clustering. ${ }^{389}$ To decrease bias, we excluded studies with a high risk of bias in the domain risk of bias arising from the randomisation process. ${ }^{40}$

\section{Search strategy}

Electronic searches

The following databases will be searched for published studies: AMED, CINAHL, EMBASE, the Cochrane Library and MEDLINE (including MEDLINE Epub Ahead of Print, In-Process and Other Non-Indexed Citations, MEDLINE Daily and MEDLINE). Unpublished and ongoing studies will be searched from WHO International Clinical Trials Registry Platform (http://www.who. int/ictrp/en/) and the US National Institutes of Health (https://clinicaltrials.gov/). Only English studies will be included and no restriction will be set for publication status. The search strategy for MEDLINE is provided as online supplementary material.

\section{Reference lists and other sources}

Reference lists of all included studies, relevant systematic reviews and meta-analyses, and guidelines will be screened for eligible additional studies to be included.

\section{Identification and selection of studies}

Two reviewers will independently screen titles and abstracts of the articles from the search. Before formal screening of titles, we will perform an intratester agreement test (kappa test) by randomly selecting 50 citations (through random number table) to be reviewed by two independent reviewers. ${ }^{38}$ An agreement of $80 \%$ or more will be considered acceptable. If we do not achieve the percentage of the agreement, we will randomly select another 50 citations subsequently until $80 \%$ of agreement is reached. Any disagreement will be solved by discussion and if necessary, a third reviewer will arbitrate the decision. When studies fail to provide the necessary data, the authors will be contacted and further information requested.

\section{Data extraction}

Two reviewers will independently extract data from the included studies using a standardised data extraction form. Similarly, a pilot test will be performed before the formal extraction. We will randomly select five articles using a random number table to confirm we have enough inter-rater agreement (at least 80\%). Any disagreement will be solved by discussion. Otherwise, a third reviewer will make a decision. The following data will be extracted from each included study based on recommendations from previous studies. ${ }^{34} 41$

1. Study characteristics, such as year of study publication, first author, journal, sample size, study funding and location.

2. Patient characteristics, such as age, gender, including and excluding criteria, diagnostic criteria, type of lumbar spinal stenosis, comorbidities, duration of symptoms and previous treatment.

3. Intervention characteristics.

4. Primary and secondary outcomes.

\section{Measurement of treatment effect}

Relative treatment effects

1. Continuous outcomes: If the studies use the same rating scale, we will use mean difference (MD) with its 95\% CI. If different rating scales are used, standardised MD with its $95 \%$ CI will be used.

2. Dichotomous outcomes: OR with its $95 \%$ CI will be used.

3. For all-cause mortality, the number needed to harm will be calculated. ${ }^{38}$

\section{Relative treatment ranking}

The surface under the cumulative ranking curve and mean ranks will be used to rank each intervention for each outcome. ${ }^{42}$ Rank-heat plot will be used to show the ranking results of each outcome for each intervention. ${ }^{43}$

\section{Dealing with missing outcome data and missing statistics}

For continuous outcomes, if the study only reports SE, $\mathrm{p}$ value or CI, we will convert them into $\mathrm{SD}^{38}$ If the study reports median and IQR, we will calculate SD by dividing the IQR by 1.35 and considering the median equivalent to the mean. ${ }^{38}$ If relevant information is provided in figures, we will extract the data from the graphs. If data cannot be obtained, we will contact the authors. If we do not obtain relevant data, informative missingness difference of means (IMDoM) will be used as one kind of sensitivity analysis to explore the uncertainty of our results under the missing at random assumption. ${ }^{44}$

For dichotomous outcomes, first, we will try to contact the authors to obtain data. In the absence of a response or of relevant data, informative missing ORs (IMORs) for dichotomous outcomes will be used to explore the uncertainty of our results under the missing at random assumption. $^{44}$

\section{Risk of bias assessment}

Two reviewers will independently assess the risk of bias of the included studies. Any disagreement will be solved by discussion. Otherwise, a third reviewer will make a decision. We will contact the authors to obtain further information if the third reviewer thinks it is necessary.

Revised Cochrane risk-of-bias tool for randomized trials (RoB 2) will be used to evaluate the risk of bias 
of included randomised parallel-group trials. ${ }^{40}$ The tool is composed of five domains: (1) bias arising from the randomisation process; (2) bias due to deviations from intended interventions; (3) bias due to missing outcome data; (4) bias due to missing outcome data and (5) bias in selection of the reported result. Each domain includes several signalling questions which elicit information relevant to an assessment of risk of bias. The answer option for each signalling question is: yes, probably yes, probably no, no and no information. Based on the answers of all signalling questions in one domain, we will rate the domain as low risk of bias, some concerns or high risk of bias. Finally, we will get the overall riskof-bias judgement as low risk of bias, some concerns or high risk of bias considering the risk-of-bias judgement in five domains.

For cluster-randomised trials, one more domain should be considered: bias arising from identification or recruitment of individual participants within clusters. For crossover trials, analysis issues in cross-over trials should be additionally considered.

\section{Data analysis}

The characteristics of study, patient and intervention will be summarised descriptively. We will make a narrative review for some comparisons if insufficient data are provided. Network plot will be drawn to descript the available interventions. The size of the node reflects the number of patients in each intervention. The breadth of the edge shows the number of comparisons. For efficacy and safety outcomes, pairwise and NMA will be performed.

\section{Pairwise meta-analyses}

We will perform traditional pairwise meta-analyses through random-effect model with DerSimonian and Laird inverse-variance method for every direct comparison. ${ }^{38}$ In some subgroups, we will also perform pairwise meta-analyses if NMAs could not be performed. The heterogeneity will be assessed by $\mathrm{I}^{2}$ and $\mathrm{T}^{2} .{ }^{38}$

\section{Assessment of the transitivity assumption}

The potential baseline effect modifiers (age, gender, education level, baseline physical function, smoking habit, body mass index (BMI), comorbidities and previous treatment) will be assessed to confirm they are similar among different comparisons before we perform NMAs. ${ }^{34}$ If any difference is found, we will perform meta-regression to explore the influence on the results.

\section{Network meta-analyses}

Random-effect NMAs under the frequentist framework will be performed to combine both direct and indirect comparisons. ${ }^{45}$ The heterogeneity parameter is assumed the same for each intervention. ${ }^{45}$ Prediction interval plot will be drawn to reflect the uncertainly of the results in a future study. ${ }^{46} 47$

\section{Assessment of inconsistency}

Bucher method as a local method and design-by-treatment interaction model as a global method will be used. ${ }^{48}$ If any inconsistency is found, node-splitting method will be used to explore the original of the inconsistency. ${ }^{45}$

\section{Exploring sources of heterogeneity or inconsistency with subgroup analyses and meta-regression}

For two primary outcomes (physical function and all-cause mortality), subgroup analyses and meta-regressions will be performed under the three-time categories (short term, mid term and long term) except for the analysis on duration of follow-up for long-term assessment. Subgroup analyses will be performed as follows: (1) Single-level spinal stenosis versus multiple levels, the hypothesis is that patients with multiple levels spinal stenosis might have poorer physical function and higher all-cause mortality than patients with single level; (2) Duration of follow-up for long-term assessment (eg, 1 year, 2 years and 5 years), the hypothesis is that patients who received injection therapies might have poorer physical function and higher all-cause mortality in longer duration of follow than patients who received surgical therapies; (3) Patients with versus patients without degenerative spondylolisthesis, the hypothesis is that patients with degenerative spondylolisthesis might have poorer physical function and higher all-cause mortality than patients without; (4) Type of disease: central, foraminal or lateral, the hypothesis is that patients with central lumbar spinal stenosis might have poorer physical function and higher all-cause mortality than patients with foraminal or lateral. Meta-regression will be performed as follows: (1) age; (2) percentage of the male; (3) sample size; (4) baseline physical function; (5) percentage of the smoker and (6) BMI.

\section{Sensitivity analyses}

For two primary outcomes (physical function and all-cause mortality), sensitivity analyses will be performed as follows: (1) only studies with low risk of bias; (2) studies with imputed data through either IMDoM or IMOR; (3) studies without a non-active comparison group; (4) studies without receiving commercial funding and (5) studies without unpublished data.

\section{Publication bias}

Comparison-adjusted funnel plot will be used to test the publication bias if the number of included studies is larger than $10{ }^{42}$ As described above, meta-regression procedures using sample size and effect estimates will be performed to detect the small-study effect. ${ }^{50}$

\section{Grading the evidence}

The Grading of Recommendations, Assessment, Development and Evaluations framework will be used to evaluate the quality of evidence. ${ }^{51}$ The tool includes five domains, which are study limitations, indirectness, inconsistency, imprecision and publication bias. 


\section{Statistical software}

All analyses (pairwise meta-analysis will be only performed in Stata and NMA will be performed in both Stata and R) will be performed in Stata (StataCorp. 2017. Stata Statistical Software: Version 15.1) and R (V.3.4.3. R Core Team. 2017. R: A language and environment for statistical computing. R Foundation for Statistical Computing, Vienna, Austria).

\section{Patient and public involvement}

Patients will not be involved.

\section{ETHICS AND DISSEMINATION}

We will publish the research in a peer-reviewed journal after completing it.

Contributors All authors conceived the study. LC drafted the manuscript. LC and PB participated in the search strategy development. PF, PB and MF assisted in protocol design and revision. All authors read and approved the final manuscript as submitted.

Funding MLF is supported by the National Health and Medical Research Council of Australia Career Development Fellowship. Other authors have not declared a specific grant for this research from any funding agency in the public, commercial or not-for-profit sectors.

Competing interests None declared.

Patient consent for publication Not required.

Ethics approval This research does not require ethics approval because it uses data from literatures.

Provenance and peer review Not commissioned; externally peer reviewed.

Open access This is an open access article distributed in accordance with the Creative Commons Attribution Non Commercial (CC BY-NC 4.0) license, which permits others to distribute, remix, adapt, build upon this work non-commercially, and license their derivative works on different terms, provided the original work is properly cited, appropriate credit is given, any changes made indicated, and the use is non-commercial. See: http://creativecommons.org/licenses/by-nc/4.0/.

\section{REFERENCES}

1. Hartvigsen J, Hancock MJ, Kongsted A, et al. What low back pain is and why we need to pay attention. Lancet 2018;391:2356-67.

2. Kreiner DS, Shaffer WO, Baisden JL, et al. An evidence-based clinical guideline for the diagnosis and treatment of degenerative lumbar spinal stenosis (update). Spine J 2013;13:734-43.

3. Kalichman L, Cole R, Kim DH, et al. Spinal stenosis prevalence and association with symptoms: the Framingham Study. Spine $J$ 2009;9:545-50.

4. Machado GC, Ferreira PH, Yoo RI, et al. Surgical options for lumbar spinal stenosis. Cochrane Database Syst Rev 2016;11:Cd012421.

5. May $\mathrm{S}$, Comer $\mathrm{C}$. Is surgery more effective than non-surgical treatment for spinal stenosis, and which non-surgical treatment is more effective? A systematic review. Physiotherapy 2013;99:12-20.

6. Benyamin RM, Staats PS, MiDAS Encore I. MILD® Is an Effective Treatment for Lumbar Spinal Stenosis with Neurogenic Claudication: MiDAS ENCORE Randomized Controlled Trial. Pain Physician 2016;19:229-42.

7. Deyo RA, Martin BI, Ching A, et al. Interspinous spacers compared with decompression or fusion for lumbar stenosis: complications and repeat operations in the Medicare population. Spine 2013;38:865-72.

8. Deyo RA, Mirza SK, Martin BI, et al. Trends, major medical complications, and charges associated with surgery for lumbar spinal stenosis in older adults. JAMA 2010;303:1259-65.

9. Elsheikh NA, Amr YM. Effect of adding calcitonin to translaminar epidural steroid in degenerative lumbar spinal canal stenosis. Pain Physician 2016;19:139-46.

10. Jansson KA, Blomqvist $P$, Granath $F$, et al. Spinal stenosis surgery in Sweden 1987-1999. Eur Spine J 2003;12:535-41.
11. Manchikanti L, Knezevic NN, Boswell MV, et al. Epidural injections for lumbar radiculopathy and spinal stenosis: a comparative systematic review and meta-analysis. Pain Physician 2016;19:E365-410.

12. Försth $\mathrm{P}$, Ólafsson G, Carlsson $\mathrm{T}$, et al. A randomized, controlled trial of fusion surgery for lumbar spinal stenosis. N Engl J Med 2016;374:1413-23.

13. Ghogawala Z, Dziura J, Butler WE, et al. Laminectomy plus Fusion versus Laminectomy Alone for Lumbar Spondylolisthesis. N Engl J Med 2016;374:1424-34.

14. Shen J, Xu S, Xu S, et al. Fusion or Not for Degenerative Lumbar Spinal Stenosis: A Meta-Analysis and Systematic Review. Pain Physician 2018;21:1-8.

15. Zhao H, Duan LJ, Gao YS, et al. Comparison of two FDA-approved interspinous spacers for treatment of lumbar spinal stenosis: Superion versus X-STOP-a meta-analysis from five randomized controlled trial studies. J Orthop Surg Res 2018;13:42.

16. Zhao XW, Ma JX, Ma XL, et al. Interspinous process devices(IPD) alone versus decompression surgery for lumbar spinal stenosis(LSS): A systematic review and meta-analysis of randomized controlled trials. Int J Surg 2017;39:57-64.

17. Yavin $D$, Casha S, Wiebe $S$, et al. Lumbar fusion for degenerative disease: a systematic review and meta-analysis. Neurosurgery 2017;80:701-15.

18. Li AM, Li X, Yang Z. Decompression and coflex interlaminar stabilisation compared with conventional surgical procedures for lumbar spinal stenosis: A systematic review and meta-analysis. Int $J$ Surg 2017;40:60-7.

19. Lee $\mathrm{CH}$, Chung $\mathrm{CK}$, Sohn MJ, et al. Short limited fusion versus long fusion with deformity correction for spinal stenosis with balanced De Novo degenerative lumbar scoliosis: a meta-analysis of direct comparative studies. Spine 2017;42:E1126-32.

20. Chang W, Yuwen P, Zhu Y, et al. Effectiveness of decompression alone versus decompression plus fusion for lumbar spinal stenosis: a systematic review and meta-analysis. Arch Orthop Trauma Surg 2017; 137:637-50.

21. Phan K, Rao PJ, Ball JR, et al. Interspinous process spacers versus traditional decompression for lumbar spinal stenosis: systematic review and meta-analysis. J Spine Surg 2016;2:31-40.

22. Phan K, Mobbs RJ. Minimally invasive versus open laminectomy for lumbar stenosis: a systematic review and meta-analysis. Spine 2016;41:E91-100.

23. Overdevest GM, Jacobs W, Vleggeert-Lankamp C, et al. Effectiveness of posterior decompression techniques compared with conventional laminectomy for lumbar stenosis. The Cochrane database of systematic reviews 2015;3:Cd010036.

24. Machado GC, Ferreira PH, Harris IA, et al. Effectiveness of surgery for lumbar spinal stenosis: a systematic review and meta-analysis. PLoS One 2015;10:e0122800.

25. Liu K, Liu P, Liu R, et al. Steroid for epidural injection in spinal stenosis: a systematic review and meta-analysis. Drug Des Devel Ther 2015;9:707-16.

26. Liang L, Jiang WM, Li XF, et al. Effect of fusion following decompression for lumbar spinal stenosis: a meta-analysis and systematic review. Int J Clin Exp Med 2015;8:14615-24.

27. Lauryssen C, Jackson RJ, Baron JM, et al. Stand-alone interspinous spacer versus decompressive laminectomy for treatment of lumbar spinal stenosis. Expert Rev Med Devices 2015;12:763-9.

28. Hong P, Liu Y, Li H. Comparison of the efficacy and safety between interspinous process distraction device and open decompression surgery in treating lumbar spinal stenosis: a meta analysis. $J$ Invest Surg 2015;28:40-9.

29. Dias S, Welton NJ, Sutton AJ, et al. Evidence synthesis for decision making 1: introduction. Med Decis Making 2013;33:597-606.

30. da Costa BR, Reichenbach S, Keller N, et al. Effectiveness of non-steroidal anti-inflammatory drugs for the treatment of pain in knee and hip osteoarthritis: a network meta-analysis. Lancet 2017;390:e21-33.

31. Feng $F, X u Q$, Yan F, et al. Comparison of 7 surgical interventions for lumbar disc herniation: a network meta-analysis. Pain Physician 2017;20:E863-71.

32. Guo JR, Jin XJ, Shen HC, et al. A comparison of the efficacy and tolerability of the treatments for sciatica: a network meta-analysis. Ann Pharmacother 2017;51:1041-52.

33. Moher D, Shamseer L, Clarke M, et al. Preferred reporting items for systematic review and meta-analysis protocols (PRISMA-P) 2015 statement. Syst Rev 2015;4:1.

34. Clement RC, Welander A, Stowell C, et al. A proposed set of metrics for standardized outcome reporting in the management of low back pain. Acta Orthop 2015;86:523-33. 
35. Chapman JR, Norvell DC, Hermsmeyer JT, et al. Evaluating common outcomes for measuring treatment success for chronic low back pain. Spine 2011;36(21 Suppl):S54-68.

36. Jensen MP, Mardekian J, Lakshminarayanan M, et al. Validity of 24-h recall ratings of pain severity: biasing effects of "Peak" and "End" pain. Pain 2008;137:422-7.

37. Dakin H, Abel L, Burns R, et al. Review and critical appraisal of studies mapping from quality of life or clinical measures to EQ-5D: an online database and application of the MAPS statement. Health Qual Life Outcomes 2018;16:31.

38. The Cochrane collaboration. Cochrane handbook for systematic reviews of interventions: The Cochrane collaboration, 2011. Available from. www.handbook.cochrane.org.

39. Caddy C, Amit BH, McCloud TL, et al. Ketamine and other glutamate receptor modulators for depression in adults. Cochrane Database Syst Rev 2015:Cd011612.

40. Chandler J, McKenzie J, Boutron I, Welch V, et al. eds. Cochrane methods: Cochrane Database of Systematic Reviews, 2016.

41. Furlan AD, Malmivaara A, Chou R, et al. 2015 Updated Method Guideline for Systematic Reviews in the Cochrane Back and Neck Group. Spine 2015;40:1660-73.

42. Salanti G, Ades AE, loannidis JP. Graphical methods and numerical summaries for presenting results from multiple-treatment metaanalysis: an overview and tutorial. J Clin Epidemiol 2011;64:163-71.
43. Veroniki AA, Straus SE, Fyraridis A, et al. The rank-heat plot is a novel way to present the results from a network meta-analysis including multiple outcomes. J Clin Epidemiol 2016;76:193-9.

44. Mavridis D, White IR, Higgins JP, et al. Allowing for uncertainty due to missing continuous outcome data in pairwise and network metaanalysis. Stat Med 2015;34:721-41.

45. White IR. Network meta-analysis. Stata J 2015;15:951-85.

46. Riley RD, Higgins JP, Deeks JJ. Interpretation of random effects meta-analyses. BMJ 2011;342:d549.

47. Higgins JP, Thompson SG, Spiegelhalter DJ. A re-evaluation of random-effects meta-analysis. J R Stat Soc Ser A Stat Soc 2009;172:137-59.

48. Bucher HC, Guyatt GH, Griffith LE, et al. The results of direct and indirect treatment comparisons in meta-analysis of randomized controlled trials. J Clin Epidemiol 1997;50:683-91.

49. Higgins JP, Jackson D, Barrett JK, et al. Consistency and inconsistency in network meta-analysis: concepts and models for multi-arm studies. Res Synth Methods 2012;3:98-110.

50. Chaimani A, Salanti G. Using network meta-analysis to evaluate the existence of small-study effects in a network of interventions. Res Synth Methods 2012;3:161-76.

51. Salanti G, Del Giovane C, Chaimani A, et al. Evaluating the quality of evidence from a network meta-analysis. PLoS One 2014;9:e99682. 